\title{
Genotypic characterization and genome comparison reveal insights into potential vaccine coverage and genealogy of Neisseria meningitidis in military camps in Vietnam
}

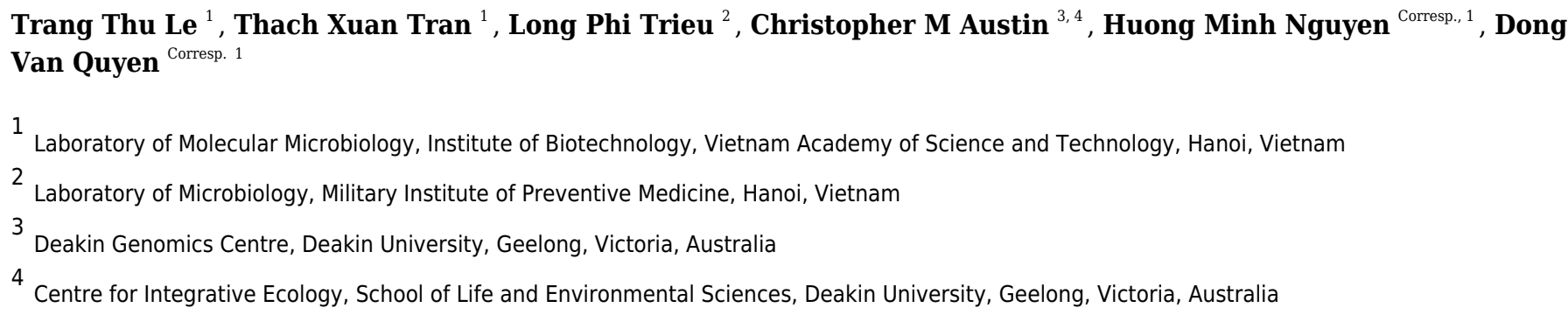

Background. Neisseria meningitidis remains the main cause of sporadic meningitis and sepsis in military camps in Vietnam. Yet, very limited molecular data of their genotypic and epidemiological characteristics are available from Vietnam, and particularly the military environment. Whole genome sequencing (WGS) has proven useful for meningococcal disease surveillance and guiding preventative vaccination programs. Previously, we characterized key genetic and epidemiological features of an invasive $N$. meningitidis $B$ isolate from a military unit in Vietnam. Here, we extend these findings by sequencing two additional invasive $N$. meningitidis $B$ isolated from cerebrospinal fluid (CSF) of two meningitis cases at another military unit and compared their genomic sequences and features. We also report the sequence types and antigenic profiles of 25 historical and more recently emerged $N$. meningitidis isolates from these units and other units in proximity. Methods. Strains were sequenced using the Illumina HiSeq platform, de novo assembled and annotated. Genomes were compared within and between military units, as well as against the global $N$. meningitidis collection and other isolates from the Southeast Asia region using PubMLST. Variations at the nucleotide level were determined, and phylogenetic relationships were estimated. Antigenic genotypes and vaccine coverage were analyzed using gMATS and PubMLST. Susceptibility of isolates against commonly used antibiotic agents was examined using E-test. Results. Genome comparison revealed a high level of similarity among isolates both within and between units. All isolates showed resistance to chloramphenicol and carried identical catP gene with other Southeast Asian isolates, suggesting a common lineage. Their antigenic genotypes predicted no coverage by either Bexsero ${ }^{\circledR}$ or Trumenba ${ }^{\circledR}$, and nucleotide variation analysis revealed diverse new, unassigned alleles at multiple virulence loci of all strains. Groups of singleton and 
unique novel sequence types extending beyond individual camps were found from epidemiological data of 25 other isolates. Our results add to the sparse published molecular data of $N$. meningitidis in the military units in Vietnam, highlight their diversity, distinct genetic features and antibiotic resistance pattern, and emphasize the need for further studies on the molecular characteristics of $N$. meningitidis in Vietnam. 
2 Genotypic characterization and genome comparison reveal insights into potential vaccine

3 coverage and genealogy of Neisseria meningitidis in military camps in Vietnam

4

5 Trang Thu Le ${ }^{1}$, Thach Xuan Tran ${ }^{1}$, Long Phi Trieu ${ }^{2}$, Christopher M. Austin ${ }^{3,4}$, Huong Minh

6 Nguyen $^{1 *}$, and Dong Van Quyen ${ }^{1 *}$

7

$8{ }^{1}$ Laboratory of Molecular Microbiology, Institute of Biotechnology, Vietnam Academy of

9 Science and Technology, Hanoi, Vietnam

$10{ }^{2}$ Laboratory of Microbiology, Military Institute of Preventive Medicine, Hanoi, Vietnam

$11{ }^{3}$ Deakin Genomics Centre, Deakin University, Geelong 3220, Australia

$12{ }^{4}$ Centre for Integrative Ecology, School of Life and Environmental Sciences, Deakin University, 13 Geelong 3220, Victoria, Australia (dvquyen@ibt.ac.vn)

Address: Laboratory of Molecular Microbiology, Institute of Biotechnology, Vietnam Academy of Science and Technology, 18 Hoang Quoc Viet, Cau Giay, Ha Noi, Viet Nam 
22

\section{ABSTRACT}

Background. Neisseria meningitidis remains the main cause of sporadic meningitis and sepsis in military camps in Vietnam. Yet, very limited molecular data of their genotypic and epidemiological characteristics are available from Vietnam, and particularly the military environment. Whole genome sequencing (WGS) has proven useful for meningococcal disease surveillance and guiding preventative vaccination programs. Previously, we characterized key genetic and epidemiological features of an invasive N. meningitidis B isolate from a military unit in Vietnam. Here, we extend these findings by sequencing two additional invasive $N$. meningitidis $\mathrm{B}$ isolated from cerebrospinal fluid (CSF) of two meningitis cases at another military unit and compared their genomic sequences and features. We also report the sequence types and antigenic profiles of 25 historical and more recently emerged $N$. meningitidis isolates from these units and other units in proximity.

Methods. Strains were sequenced using the Illumina HiSeq platform, de novo assembled and annotated. Genomes were compared within and between military units, as well as against the global N. meningitidis collection and other isolates from the Southeast Asia region using PubMLST. Variations at the nucleotide level were determined, and phylogenetic relationships were estimated. Antigenic genotypes and vaccine coverage were analyzed using gMATS and PubMLST. Susceptibility of isolates against commonly used antibiotic agents was examined using E-test.

Results. Genome comparison revealed a high level of similarity among isolates both within and between units. All isolates showed resistance to chloramphenicol and carried identical catP gene with other Southeast Asian isolates, suggesting a common lineage. Their antigenic genotypes 
44 predicted no coverage by either Bexsero ${ }^{\circledR}$ or Trumenba ${ }^{\circledR}$, and nucleotide variation analysis

45 revealed diverse new, unassigned alleles at multiple virulence loci of all strains. Groups of

46 singleton and unique novel sequence types extending beyond individual camps were found from

47 epidemiological data of 25 other isolates. Our results add to the sparse published molecular data

48 of N. meningitidis in the military units in Vietnam, highlight their diversity, distinct genetic

49 features and antibiotic resistance pattern, and emphasize the need for further studies on the

50 molecular characteristics of $N$. meningitidis in Vietnam.

INTRODUCTION

53

54

55

56

57

58

59

60

61

62

63

64

65

66

Neisseria meningitidis is an encapsulated Gram-negative bacterium that

asymptomatically colonizes the human nasopharynx but can cause serious septicemia and meningitis upon entering the blood stream and passing through the blood-meningeal barrier (Rosenstein et al. 2001). Carriage rate is age and setting dependent, among other factors, with high prevalence found in the age groups of infants (4.5\%) and young adults $(23.7 \%)$ (Christensen et al. 2010). Congregated living environment is another risk factor, as shown in increased carriage rate among university students living in dormitories (Peterson et al. 2018; Breakwell et al. 2018) or military recruits in camp sites (Sim et al. 2013; Keiser, Hamilton, and Broderick 2011). In these environments, meningococcal meningitis can sometimes become an outbreak, and sporadic cases often show recurring and cluster characteristics (Peterson et al. 2018). Hence, in many countries, preventative vaccination program is recommended for these high-risk groups (Yezli, Wilder-Smith, and Bin Saeed 2016) and implementations have shown significant impact (Broderick, Phillips, and Faix 2015). However, to enable successful preventative implementation and reduce the risk related to these environments, as well as to aid future cases' diagnosis and 
67 treatment, it is crucial to have reliable and accurate monitoring data of sporadic cases and

68

69

70

71

72

73

74

75

76

77

78

79

80

81

82

83

84

85

carriages in these groups.

Recent advances in genome sequencing technology allows for a significant volume of genomic data to be generated and made public. Such data also provide an unprecedented power of discrimination that is invaluable for studies of the relationship of closely related strains. The widely utilized Neisseria database PubMLST (https://pubmlst.org/neisseria/), at the time of preparation of this manuscript, hosts more than 22,000 genomes and 60,000 isolate records for Neisseria. Employing sequence data deposited to PubMLST, a core genome of 1605 loci was determined for N. meningitidis (Bratcher et al. 2014). Analysis of sequence variations at these loci has furthered our understanding of genomic variation within N. meningitidis population (Harrison et al. 2017) and enabled the study of closely related but distinct strains present in outbreaks (Jolley et al. 2012). Despite the usefulness of whole genome sequencing (WGS) analysis for epidemiological surveillance, data from Vietnam, and Southeast Asian region in general, are extremely limited. Previously, we have described for the first time the genome of a chloramphenicol-resistant invasive $N$. meningitidis B isolated from a military unit in Vietnam (Tran et al. 2019). Later, a study conducted by the Mahidol-Oxford Tropical Health Network (MORU) in Thailand, Laos and Cambodia identified eight additional N. meningitidis isolates carrying the identical chloramphenicol-resistant gene along other acquired resistance to multiple antibiotics (Batty et al. 2019), suggesting the existence and expansion of a lineage in this region. Here we extend the previous studies by describing the genomic characteristics of two additional invasive $N$. meningitidis B isolated from CSF of two meningitis cases at another Vietnamese military unit in proximity with the previously reported, and conduct a global analysis utilizing the PubMLST database and associated analytical tools. We show that besides the 
90

91

92

93

94

95

96

97

chloramphenicol non-susceptible lineage, reservoirs of known sequence types that cannot be assigned to lineages, novel emerging sequence types make up a significant part of both invasive and carriage strains found in the military camp environments in Vietnam.

\section{MATERIALS AND METHODS}

\section{Bacterial isolation and typing}

All invasive $N$. meningitidis strains were isolated from CSF and all carrier strains were isolated from mouthwash samples at the Laboratory of Microbiology, Military Institute of Preventive Medicine, Hanoi as described previously (Tran et al. 2019). Two isolates, NMB_VN2013 and NMB_VN2015, were from the CSF of two confirmed meningitis cases discovered in 2013 and 2015 at Military Unit 2, a camp in the geographical closeness to Military Unit 1, the camp where the previously reported DuyDNT isolate were identified. Both cases were treated at the military hospital and subsequently recovered. Serogroup identification and multi-locus sequence typing (MLST) were either done according to previously described standard methods (Organization 2011) or by manually extracting the corresponding sequences from WGS data.

DuyDNT isolate was renamed NMB_VN2014 here for convenience and consistency. Available laboratory records of the Laboratory of Microbiology, Military Institute of Preventive Medicine, Hanoi were reviewed and a suitable collection of 25 isolates were chosen based on reported year (before and after 2013 - 2015), location (Military Unit 1, 2, and two nearby units here named Unit 3 and Unit 4) and availability of molecular characterization data (Table 1). Serogroup data, MLST data, $f H b p$ and porA allelic variants were obtained from laboratory 
112 records. Case-related metadata, including year, clinical status, and location were obtained where

113 available.

114 Antibiotic susceptibility testing

115 Susceptibility of isolates to seven antibiotics, namely ampicillin, ciprofloxacin,

116 cefotaxime, ceftriaxone, rifampicin, meropenem and chloramphenicol, was examined using E-

117 test strip (bioMerieux, France) following manufacturer's guideline and MIC values were

118 determined. Susceptibility was interpreted according to CLSI 2018 breakpoints (CLSI 2018).

119 Genome sequencing and analysis

120 Genomic DNA was extracted using GeneJET Genomic DNA Purification Kit

121 (Thermofisher Scientific) in accordance with the manufacturer's instruction. Samples' quality

122 was checked before sequenced using the Illumina HiSeq 4000 system (Macrogen). Genome

123 assembly and annotation were performed as previously described (Tran et al. 2019). Annotated

124 amino acid sequences were used to identify genes involved in antibiotic resistance (Tran et al.

125 2019). Additionally, allelic profile of relevant antibiotic resistant genes from PubMLST were

126 extracted from WGS data of each genome, and where applied, PSI-BLAST were used to find

127 homologous sequence. Antigenic profile, antibody cross reactivity prediction and allelic variants

128 of virulent factors including capsular genes, Maf-toxin island, and outer membrane vesicle

129 (OMV) genes were analyzed with gMATS (Muzzi et al. 2019) and the PubMLST server using

130 default parameters.

131 Genome comparison and phylogenetic analysis

132 Assembled genomes were submitted to the PubMLST website and allelic variants were

133 automatically assigned for each locus. Genomes were compared at the seven loci of MLST 
134 scheme, the 53 loci of ribosomal MLST scheme, and the 1605 loci of the core genome cgMLST

135 scheme for N. meningitidis. Allelic variants were further processed in Excel and where

136 necessary, manually removed from comparisons. Genomes were aligned using the progressive

137 Mauve software (Darling, Mau, and Perna 2010), and the phylogenetic distance between strains

138 was determined at the whole genome level. Neighbor-Net networks were constructed from

139 various comparisons implemented on the PubMLST and visualized by SplitsTree (Huson 1998).

140 Raw sequence data of 18 Southeast Asian N. meningitidis was obtained from the

141 European Nucleotide Archive (project PRJEB30968) (Batty et al. 2019), assembled using Spades

142 V3.11.1 (Bankevich et al. 2012), and assembled contigs were used for phylogenetic network

143 analysis by SplitsTree and other sequence comparisons.

RESULTS AND DISCUSSION

\section{Characterization of NMB_VN2013 and NMB_VN2015 isolates}

Genome content, and contained 2390 and 2409 CDS each, respectively. Each genome had 3 rRNA and 53 tRNA coding sequences and contained $\sim 400$ repetitive sequences. Overall, their genome size and content matched closely with the typical genome of previously reported Neisseria representatives such as N. meningitidis MC58 (Tettelin et al. 2000), Z2491 (Parkhill et al. 2000), FAM18 (Bentley et al. 2007), and N. gonorrhoeae NCCP11945 (Chung et al. 2008). Assembly

154 data and genomic sequences of both genomes were deposited to NCBI Genomes database under 155 BioProject ID PRJNA523495. 
156

157

158

159

160

161

162

163

164

165

166

167

168

169

170

171

172

173

174

175

176

177

178

\section{Serogroup and Sequence type}

Both NMB_VN2013 and NMB_VN2015 were serogroup B, as inferred by the presence of the $c s b$ gene from their genome sequence and confirmed by Vitek ${ }^{\circledR}$. Multi-locus sequence typing (MLST) profiles extracted from WGS data grouped NMB_VN2013 and NMB_VN2015 into the same ST-1576, a singleton ST that had no known clonal complex. ST-1576 is closely related to ST-13074, which was assigned to NMB_VN2014 before, with two STs differed at a single locus (aroE). Polymorphic site analysis revealed 46 nucleotide changes and no deletion/insertion between the two alleles, aroE 9 of ST-1576 and aroE 4 of ST-13074.

\section{Antibiotic susceptibility}

Previously, NMB_VN2014 was shown to carry a tetracycline (rps $)$ and chloramphenicol (catP) resistant genes. Identical rps $J$ and catP genes were found in the genomes of NMB_VN2013 and NMB_VN2015. The 624 bp catP gene found in all three Vietnamese isolates was the same gene previously reported in France (Galimand et al. 1998), and Southeast Asia (Batty et al. 2019). Antibiotic susceptibility test confirmed NMB_VN2013 and NMB_VN2015's resistance to chloramphenicol, with the recorded MIC were 62 and $64 \mu \mathrm{g} / \mathrm{ml}$, respectively (Table 2).

From WGS data, of the 11 antibiotic susceptibility genes analyzed by PubMLST, eight were identical in all isolates, including gyrA (allele 2), pen $A$ (allele 587) and $r p o B$ (allele 42). Both gyrA 2 and rpoB 42 alleles were previously shown to confer no resistance to ciprofloxacin and rifampicin, respectively (Hong et al. 2013; Taha et al. 2007). Loci NEIS1609 (folP) from all isolates, NEIS1600 (parE) from NMB_VN2014, and NEIS1753 (penA) from NMB_VN2013 and NMB_VN2014 had new allelic variants with no assigned numbers. NEIS1600 and NEIS1753, together with NEIS1635 ( $m$ trR $)$, represented three variable loci among the isolates of 
179 this study (Table 2). Notably, both allelic variants at $m$ trR locus (7 in NMB_VN2013 and

180 NMB_VN2015, and 1086 in NMB_VN2014) harbored the A39T mutation. This mutation was

181 observed significantly more often in azithromycin-exposed N. gonorrhoea carriers (Wind et al.

182 2017), and may result in overexpression of the MtrCDE efflux pump and increased antibiotic

183 resistance in $N$. gonorrhoea (Demczuk et al. 2017). Although azithromycin is not recommended

184 by the Vietnam Ministry of Health for treatment of meningitis, it is recommended by the WHO

185 for the dual therapy (along with ceftriaxone) to treat $N$. gonorrhoea infection. It is thus important

186 to monitor the presence and spreading to potential azithromycin-resistant genetic features in

187 Neisseria genus. Other well-known azithromycin-resistant mutations, 23S rRNA A2045G and

188 C2597T, first identified in N. gonorrhoea (Demczuk et al. 2017), were not found in any

189 Vietnamese isolates in this study which all carried wild-type $23 \mathrm{~S}$ rRNA.

We confirmed antibiotic susceptibility of NMB_VN2013 and NMB_VN2015 by MIC

191 test, and both strains showed sensitivity to ciprofloxacin, rifampicin, cefotaxime, and

192 ceftriaxone; but diminished susceptibility toward ampicillin, and resistance toward

chloramphenicol, though the recorded MICs were much lower than that of NMB_VN2014.

While NMB_VN2014 showed reduced susceptibility to rifampicin (MIC $=1.5 \mu \mathrm{g} / \mathrm{ml})$, both

NMB_VN2013 and NMB_VN2015 were still susceptible $(\mathrm{MIC}=0.25$ and $0.125 \mu \mathrm{g} / \mathrm{ml}$, respectively) (Table 2).

197

200

201

\section{Antigenic profiles}

Analysis of the deduced peptide sequence at antigenic loci using PubMLST showed all three Vietnamese isolate's genomes carried FHbp Peptide 31, NhbA Peptide 16, and no NadA peptide, but different por $A$ and $f e t A$ variants (Table 3). In detail, NMB_VN2014 and

NMB_VN2015 both had porA VR1 22-25, but different porA VR2, 14-32 and 14, respectively. 
202 NMB_VN2013 carried distinct porA variants (VR1 7-2, VR2 13) but shared the same fetA 203 variant (F4-6) with NMB_VN2014. NMB-VN2015 carried fetA variant 1-7. FHbp Peptide 31

204 belonged to subfamily A, 34 amino acid substituted from Peptide 19 (Trumenba®vaccine 2014) 205 and 97 amino acid differed from Peptide 1 (BEXSERO®vaccine 2015). NhbA Peptide 16

206 contained 79 amino acid substitutions from the Bexsero® component NhbA (Peptide 2). Allele

207 22-25 of VR1 region of porA had eight amino acid substitutions and one deletion compared to 208 allele7-2, and both were not the 1.4 variant used in Bexsero®.

When compared with clinical profiles of the Southeast Asian isolates reported recently by Batty and colleagues (Batty et al. 2019), a chloramphenicol-resistant lineage specific features could be observed in sequence types, FHbp Peptide, and NhbA Peptide variants of the Vietnamese and the chloramphenicol-resistant Southeast Asian isolates (Table 3). On the other hand, fet $A$ variants showed more variables among groups, and the Vietnamese porA loci shared variants with the chloramphenicol-susceptible groups instead of the resistant group. of 4 CMenB (Bexsero ${ }^{\circledR}$ ) based on $f H b p$, $n h b A$, and porA VR2 specific genotypes (Muzzi et al. 2019), all three Vietnamese isolates were $f H b p$ and por $A$ non-coverage and $n h b A$ unpredictable. components (NM03, NM14, and NM15) while one (NM23) was covered by just $n h b A$. among 21 isolates, respectively. Non-coverage was predicted at $71.4 \%, 19 \%$, and $85.7 \%$ at $f H b p$, $n h b A$, and $\operatorname{por} A$, respectively. The rest of variants, including $14.3 \%$ of $f H b p, 61.9 \%$ of $n h b A$, and none of porA were unpredictable by gMATS. Considering both expression level and genotyping, 224 and extending vaccine coverage prediction to both Trumenba ${ }^{\circledR}$ and Bexsero ${ }^{\circledR}$, PubMLST 
225 antibody cross reactivity also predicted no protection of either vaccine against all three

226 Vietnamese isolates of this study.

\section{Inference of the Vietnamese isolates' genealogy}

228

229

230

231

232

Neighbor-Net analysis was used to construct the phylogenetic networks of three Vietnamese isolates and the well-studied 108-isolate global N. meningitidis collection (Bratcher et al. 2014) based on the seven-locus (MLST), 53-locus (rMLST) and 1605-locus (cgMLST) comparisons. This collection represents the major hyper-invasive lineages/sub-lineages recorded worldwide in the last 70 years. All three methods grouped the Vietnamese isolates into a welldefined, separate clade from the rest of the network. While similar relationships for the Vietnamese isolates were maintained in all analyses, the 1605-locus cgMLST analysis was able to place the Vietnamese branch at the stem of the previously defined Lineage 3 (ST-41/44 clonal complex) (Figure 1). Additionally, only cgMLST analysis could separate the Vietnamese isolates into three unique strains; while MLST comparison grouped NMB_VN2013 and NMB_VN2015 into one ST, and rMLST identified NMB_VN2013 and NMB_VN2014 as a single strain; showing the close relationship among these strains.

A more refined cluster was observed when the 18 Southeast Asian invasive $N$. meningitidis were added to the genealogical analysis. Eleven chloramphenicol-resistant isolates, including three Vietnamese isolates, four Thai isolates, and two each from Laos and Cambodia, formed a distinct group, diverged from the rest of Southeast Asian isolates. This lineage seemed to have rapidly expanded clonally in recent years, though this could partially be due to better case report and laboratory detection, since data from this region was scarce up until recently although still remains limited. Although NeighborNet analysis placed this lineage as a divergent 
247 branch from other chloramphenicol susceptible isolates in the region, due to the limited number

248 of samples, it remains a possibility for their origin.

The rest of isolates also clustered into two groups, one included NM03, NM14, and

250 NM15 that clustered to the ST-41 sub-lineage of ST-41/44 clonal complex, while the remaining

251 isolates clustered together, seemingly formed a group connecting the chloramphenicol-resistant

252 lineage with ST-44 sub-lineage of ST-41/44 clonal complex (Figure 1).

253

254

255

256

257

258

259

260

261

262

263

264

265

266

267

268

269

\section{Relationships among Vietnamese isolates revealed by genome comparisons}

Of the 1605 loci compared, Genome Comparator identified 1245 identical loci and 355 variable loci in at least one genome of the three isolates in this study. Five loci were missing in all three isolates, four of those were pseudo genes and one encoded for a phage-related protein.

Fifteen loci were paralogous loci presumably resulting from assembly of repetitive sequences and thus were excluded from the final analysis. Resultant variable loci were further examined to exclude all variations from genes/pseudo-genes encoding for hypothetical proteins.

Consequently, from the initially identified 355 variable loci, we confirmed 264 loci that contained either point mutations, insertions/deletions, or allelic replacements from the three genomes. Among these, a number of sequence variables were observed in adjacent genes, suggesting frequent recombination events between genomes. Pairwise, the genomes of NMB_VN2013 and NMB_VN2014 showed the highest similarity, and NMB_VN2014 and NMB_VN2015 pair showed the lowest. However, pairwise phylogenetic distance between strains calculated from the progressiveMauve alignment using whole genome content was in the range of 0.0158 to 0.0112 , indicating a close relationship among them.

Besides core genome comparison, sequences of the known genetic determinants for the virulence of invasive $N$. meningitidis, namely the capsular gene cluster, the Maf-toxin island, and 
270 genes encoding for the outer membrane vesicle (OMV) peptides and pilin, from the genomes of

271 three isolates were also compared. The resulting differences, after excluding all variables due to

272 assembly or in the coding sequence of hypothetical proteins, are listed in Table 4. Overall, higher

273 sequence variability could be observed among the three genomes at these loci. From 19 loci of

274 the capsular gene cluster being present for comparison, four confirmed variable loci were $r f b A$,

$275 \operatorname{css} A, \operatorname{ctrF}$, and $c n l$. Except for two assigned alleles found in two loci (allele 113, cssA locus of

276 NMB_VN2015's genome and allele 4, ctrF locus of NMB_VN2013's genome), the rest of the

277 alleles were newly identified and not yet assigned an allelic number. Four out of 45 loci of the

278 Maf-toxin genomic island included in this comparison, namely anmK, mafIo2MGI-2,

279 mafA_MGI-3, and $m a f B \_M G I-3$, appeared to be different. Sequences of the anmK locus of

280 NMB_VN2015 and the mafB_MGI-3 locus of NMB_VN2014 genome appeared to be novel,

281 unassigned alleles. Detailed nucleotide changes analysis revealed a higher percentage of changed

282 nucleotides compared to any other single locus in both mafA_MGI-3 and mafB_MGI-3, which

283 were adjacent to each other, suggesting a recombination event could have happened in this

284 region. Compared to the capsular gene cluster and Maf-toxin island, the CDS of OMV and pilin

285 genes showed higher sequence diversity with variables scattered among loci.

286

287

288

289

290

291

292

To better understand the probable evolutionary context of these strains, we have further

collected and analyzed 25 isolates representing a collection of both historical and later emerged isolates from the same or nearby military units with the three in this study (Table 1).

Epidemiological data of these 25 isolates revealed the predominance of serogroup B $N$.

meningitidis (78.5\%) and the recurrence of two major lineages centered around ST-1576 and ST4821 in all camp sites (Table 1). The predominance of serogroup B agrees with the previous report from sporadic cases in the region (Pancharoen et al. 2000). The other serogroup observed 
293 was serogroup C (21.5\%). Both ST-1576 and ST-4821 were major hyper-virulent STs with a

294 long history and worldwide distribution. Antigenic profiles associated with these major STs

295 show high similarity to strains described before in China during 1978 - 2013 (Zhu et al. 2015).

296 Besides these, strains representing singleton and novel STs were also frequently observed

297 throughout the years and locations. Available antigenic profiles suggested frequent exchange of

298 genetic material via recombination among strains and reservoirs, with several alleles (P1.20,

299 P1.7-2) associated with the major lineages frequently recurring in different combinations. Clonal

300 expansion was also observed, indicated by the emergence of novel sequence types, and showed

301 no distinct cluster in regard of geological locations, reflecting the close and frequent contact

302 nature of the training units of new military recruits, suggesting close transmission extending

303 beyond individual camps seemed to be the main driving force for $N$. meningitidis prevalence and 304 expansion within the military environment in Vietnam. From searching through the PubMLST

305 database, many emergent STs identified were limited to these units only, highlighting the niche 306 characteristic of $N$. meningitidis population of the military camps.

$N$. meningitidis remains the main cause of sporadic meningitis and sepsis in military

308 recruit camps (Tran et al. 2019; Sim et al. 2013; Keiser, Hamilton, and Broderick 2011).

309 Accurate identification and characterization of the causative strain is crucial for the success of

310 treatment for patients and prophylaxis for contact persons, as well as prevention of outbreaks.

311 Records of Vietnamese and Southeast Asian N. meningitidis isolates are still extremely limited,

312 thus it is not possible to determine the origin of these strains, or how they have arisen. Study at

313 the genomic level of additional historical invasive and carriage strains collected at these and

314 nearby camps, or nearby regions can help identify the phylogenetic routes that led to their

315 emergence. On the other hand, since the military setting is among the highest risk group for 
316 meningococcal disease in adults, continual effort is needed to provide the surveillance data

317 essential for effective policy making and preparation for response in case of potential outbreaks 318 in the future.

CONCLUSIONS

In a previous study, we described for the first time the genome of a chloramphenicolresistant invasive $N$. meningitidis B isolate from a military unit in Vietnam. In this study, using WGS analysis, we characterized the genetic features of two additional $N$. meningitidis $\mathrm{B}$ isolates causing sporadic meningitis in another military camp in Vietnam. Core genome comparisons highlight the close phylogenetic relationship of isolates both within and between camps, with emphasis on their shared antibiotic resistant genes and antigenic profiles that are likely not yet covered by current meningococcal B vaccines, Trumenba ${ }^{\circledR}$ and Bexsero ${ }^{\circledR}$. Another notable shared feature of these isolates was their high resistance against chloramphenicol, likely attributed by but not limited to the $624 \mathrm{bp}$ cat $P$ variant that were previously found in chloramphenicol-resistant isolates in France (Galimand et al. 1998) and Southeast Asia (Batty et al. 2019). A phylogenetic network reconstructed from core genome comparison suggests a common lineage of chloramphenicol resistant isolates in the military camps of Vietnam and other Southeast Asian countries that seemed to be expanding in this region. Vietnam remains limited, we also reported epidemiological analysis of 25 invasive and carriage strains from these Vietnamese military camps. Besides the major lineages, additional groups of singleton and unique novel sequence types extending beyond individual camps were observed, indicating close transmission is likely the main driving force for $N$. meningitidis prevalence and 
339 expansion within the military environment in Vietnam. Taken together, our results provide useful

340 information for further understanding the molecular epidemiology of $N$. meningitidis in the

341 military units in Vietnam, aiding future meningococcal meningitis monitoring and surveillance in

342 the country.

344 ACKNOWLEDGEMENTS

345 We thank members of our laboratories for meaningful discussion and technical assistance. This

346 research utilized the PubMLST database (https://pubmlst.org/) developed by Keith Jolley (Jolley

347 and Maiden 2010).

348 Competing interests

349 The authors declare that they have no competing interests.

350 Availability of data and materials

351 Assembly data and genomic sequences were deposited to NCBI Genomes database under

352 BioProject ID PRJNA523495. Isolate records and novel sequence types were submitted to 353 PubMLST.

354 Authors' contributions

355 TTL, TXT, and LPT performed experiments. LPT cultured isolates and provide laboratory 356 records of additional isolates. CMA supported data analysis and contributed to revising and 357 proofreading the manuscript. DVQ contributed to study design and manuscript drafting and 358 revision. HMN designed experiments, interpreted data, wrote and revised the manuscript. All 359 authors read and approved the final manuscript. 


\section{REFERENCES}

362

363

364

365

366

367

368

369

370

371

372

373

374

375

376

377

378

379

380

381

382

383

384

385

386

387

388

389

390

391

392

393

394

395

396

397

398

399

400

401

402

403

404

Bankevich, A., S. Nurk, D. Antipov, A. A. Gurevich, M. Dvorkin, A. S. Kulikov, V. M. Lesin, S. I. Nikolenko, S. Pham, A. D. Prjibelski, A. V. Pyshkin, A. V. Sirotkin, N. Vyahhi, G. Tesler, M. A. Alekseyev, and P. A. Pevzner. 2012. 'SPAdes: a new genome assembly algorithm and its applications to single-cell sequencing', J Comput Biol, 19: 455-77.

Batty, Elizabeth M, Tomas-Paul Cusack, Janjira Thaipadungpanit, Wanitda Watthanaworawit, Verena Carrara, Somsavanh Sihalath, Jill Hopkins, Sona Soeng, Clare Ling, and Paul Turner. 2019. 'The spread of chloramphenicol-resistant Neisseria meningitidis in Southeast Asia', BioRxiv.

Bentley, S. D., G. S. Vernikos, L. A. Snyder, C. Churcher, C. Arrowsmith, T. Chillingworth, A. Cronin, P. H. Davis, N. E. Holroyd, K. Jagels, M. Maddison, S. Moule, E. Rabbinowitsch, S. Sharp, L. Unwin, S. Whitehead, M. A. Quail, M. Achtman, B. Barrell, N. J. Saunders, and J. Parkhill. 2007. 'Meningococcal genetic variation mechanisms viewed through comparative analysis of serogroup C strain FAM18', PLoS Genet, 3: e23.

BEXSERO ${ }^{\circledR}$ vaccine. 2015. 'Novartis Vaccines and Diagnostics. Bexsero: highlights of prescribing information. $\quad$ [cited $2016 \quad$ Mar http://www.fda.gov/downloads/BiologicsBloodVaccines/Vaccines/ApprovedProducts/UCM4314 47.pdf.

Bratcher, H. B., C. Corton, K. A. Jolley, J. Parkhill, and M. C. Maiden. 2014. 'A gene-by-gene population genomics platform: de novo assembly, annotation and genealogical analysis of 108 representative Neisseria meningitidis genomes', BMC genomics, 15: 1138.

Breakwell, L., M. Whaley, U. I. Khan, U. Bandy, N. Alexander-Scott, L. Dupont, C. Vanner, H. Y. Chang, J. T. Vuong, S. Martin, J. R. MacNeil, X. Wang, and S. A. Meyer. 2018. 'Meningococcal carriage among a university student population - United States, 2015', Vaccine, 36: 29-35.

Broderick, M. P., C. Phillips, and D. Faix. 2015. 'Meningococcal disease in US military personnel before and after adoption of conjugate vaccine', Emerg Infect Dis, 21: 377-9.

Christensen, H., M. May, L. Bowen, M. Hickman, and C. L. Trotter. 2010. 'Meningococcal carriage by age: a systematic review and meta-analysis', Lancet Infect Dis, 10: 853-61.

Chung, G. T., J. S. Yoo, H. B. Oh, Y. S. Lee, S. H. Cha, S. J. Kim, and C. K. Yoo. 2008. 'Complete genome sequence of Neisseria gonorrhoeae NCCP11945', J Bacteriol, 190: 6035-6.

CLSI. 2018. M100 Performance Standards for Antimicrobial Susceptibility Testing (Clinical and Laboratory Standards Institute ).

Darling, A. E., B. Mau, and N. T. Perna. 2010. 'progressiveMauve: multiple genome alignment with gene gain, loss and rearrangement', PloS one, 5: e11147.

Demczuk, W., S. Sidhu, M. Unemo, D. M. Whiley, V. G. Allen, J. R. Dillon, M. Cole, C. Seah, E. Trembizki, D. L. Trees, E. N. Kersh, A. J. Abrams, H. J. C. de Vries, A. P. van Dam, I. Medina, A. Bharat, M. R. Mulvey, G. Van Domselaar, and I. Martin. 2017. 'Neisseria gonorrhoeae Sequence Typing for Antimicrobial Resistance, a Novel Antimicrobial Resistance Multilocus Typing Scheme for Tracking Global Dissemination of N. gonorrhoeae Strains', J Clin Microbiol, 55: 1454-68.

Galimand, M., G. Gerbaud, M. Guibourdenche, J. Y. Riou, and P. Courvalin. 1998. 'High-level chloramphenicol resistance in Neisseria meningitidis', N Engl J Med, 339: 868-74.

Harrison, O. B., C. Schoen, A. C. Retchless, X. Wang, K. A. Jolley, J. E. Bray, and M. C. J. Maiden. 2017. 'Neisseria genomics: current status and future perspectives', Pathog Dis, 75.

Hong, E., S. Thulin Hedberg, R. Abad, C. Fazio, R. Enriquez, A. E. Deghmane, K. A. Jolley, P. Stefanelli, M. Unemo, J. A. Vazquez, F. J. Veyrier, and M. K. Taha. 2013. 'Target gene sequencing to define the

Peer) reviewing PDF | (2020:03:46409:2:0:NEW 6 Jun 2020) 
411

412

413

414

415

416

417

418

419

420

421

422

423

424

425

426

427

428

429

430

431

432

433

434

435

436

437

438

439

440

441

442

443

444

445

446

447

448

449

450

susceptibility of Neisseria meningitidis to ciprofloxacin', Antimicrob Agents Chemother, 57: 19614.

Huson, D. H. 1998. 'SplitsTree: analyzing and visualizing evolutionary data', Bioinformatics, 14: 68-73.

Jolley, K. A., D. M. Hill, H. B. Bratcher, O. B. Harrison, I. M. Feavers, J. Parkhill, and M. C. Maiden. 2012. 'Resolution of a meningococcal disease outbreak from whole-genome sequence data with rapid Web-based analysis methods', J Clin Microbiol, 50: 3046-53.

Jolley, K. A., and M. C. Maiden. 2010. 'BIGSdb: Scalable analysis of bacterial genome variation at the population level', BMC Bioinformatics, 11: 595.

Keiser, Paul B, Lanette Hamilton, and Michael Broderick. 2011. 'US military fatalities due to Neisseria meningitidis: case reports and historical perspective', Military medicine, 176: 308-11.

Muzzi, A., A. Brozzi, L. Serino, M. Bodini, R. Abad, D. Caugant, M. Comanducci, A. P. Lemos, M. C. Gorla, P. Krizova, C. Mikula, R. Mulhall, M. Nissen, H. Nohynek, M. J. Simoes, A. Skoczynska, P. Stefanelli, M. K. Taha, M. Toropainen, G. Tzanakaki, K. Vadivelu-Pechai, P. Watson, J. A. Vazquez, G. Rajam, R. Rappuoli, R. Borrow, and D. Medini. 2019. 'Genetic Meningococcal Antigen Typing System (gMATS): A genotyping tool that predicts 4CMenB strain coverage worldwide', Vaccine, 37: 9911000.

Organization, World Health. 2011. 'Laboratory methods for the diagnosis of meningitis caused by Neisseria meningitidis, Streptococcus pneumoniae, and Haemophilus infuenzae.' in, WHO Manual.

Pancharoen, C., S. Hongsiriwon, K. Swasdichai, T. Puthanakit, A. Tangsathapornpong, S. Lolekha, W. Punpanich, U. Tarunotai, B. Warachit, J. Mekmullica, P. Kosalaraksa, K. Chokephaibulkit, and A. Kerdpanich. 2000. 'Epidemiology of invasive meningococcal disease in 13 government hospitals in Thailand, 1994-1999', Southeast Asian J Trop Med Public Health, 31: 708-11.

Parkhill, J., M. Achtman, K. D. James, S. D. Bentley, C. Churcher, S. R. Klee, G. Morelli, D. Basham, D. Brown, T. Chillingworth, R. M. Davies, P. Davis, K. Devlin, T. Feltwell, N. Hamlin, S. Holroyd, K. Jagels, S. Leather, S. Moule, K. Mungall, M. A. Quail, M. A. Rajandream, K. M. Rutherford, M. Simmonds, J. Skelton, S. Whitehead, B. G. Spratt, and B. G. Barrell. 2000. 'Complete DNA sequence of a serogroup A strain of Neisseria meningitidis Z2491', Nature, 404: 502-6.

Peterson, M. E., R. Mile, Y. Li, H. Nair, and M. H. Kyaw. 2018. 'Meningococcal carriage in high-risk settings: A systematic review', Int J Infect Dis, 73: 109-17.

Rosenstein, N. E., B. A. Perkins, D. S. Stephens, T. Popovic, and J. M. Hughes. 2001. 'Meningococcal disease', N Engl J Med, 344: 1378-88.

Sim, S. H., J. Y. Heo, E. C. Kim, and K. W. Choe. 2013. 'A case of meningococcal sepsis and meningitis with complement 7 deficiency in a military trainee', Infect Chemother, 45: 94-8.

Taha, M. K., J. A. Vazquez, E. Hong, D. E. Bennett, S. Bertrand, S. Bukovski, M. T. Cafferkey, F. Carion, J. J. Christensen, M. Diggle, G. Edwards, R. Enriquez, C. Fazio, M. Frosch, S. Heuberger, S. Hoffmann, K. A. Jolley, M. Kadlubowski, A. Kechrid, K. Kesanopoulos, P. Kriz, L. Lambertsen, I. Levenet, M. Musilek, M. Paragi, A. Saguer, A. Skoczynska, P. Stefanelli, S. Thulin, G. Tzanakaki, M. Unemo, U. Vogel, and M. L. Zarantonelli. 2007. 'Target gene sequencing to characterize the penicillin G susceptibility of Neisseria meningitidis', Antimicrob Agents Chemother, 51: 2784-92.

Tettelin, H., N. J. Saunders, J. Heidelberg, A. C. Jeffries, K. E. Nelson, J. A. Eisen, K. A. Ketchum, D. W. Hood, J. F. Peden, R. J. Dodson, W. C. Nelson, M. L. Gwinn, R. DeBoy, J. D. Peterson, E. K. Hickey, D. H. Haft, S. L. Salzberg, O. White, R. D. Fleischmann, B. A. Dougherty, T. Mason, A. Ciecko, D. S. Parksey, E. Blair, H. Cittone, E. B. Clark, M. D. Cotton, T. R. Utterback, H. Khouri, H. Qin, J. Vamathevan, J. Gill, V. Scarlato, V. Masignani, M. Pizza, G. Grandi, L. Sun, H. O. Smith, C. M. Fraser, E. R. Moxon, R. Rappuoli, and J. C. Venter. 2000. 'Complete genome sequence of Neisseria meningitidis serogroup B strain MC58', Science, 287: 1809-15. 
451 Tran, T. X., T. T. Le, L. P. Trieu, C. M. Austin, D. Van Quyen, and H. M. Nguyen. 2019. 'Whole-genome

452

453

454

455

456

457

458

459

460

461

462

463

464

465 sequencing and characterization of an antibiotic resistant Neisseria meningitidis $\mathrm{B}$ isolate from a military unit in Vietnam', Ann Clin Microbiol Antimicrob, 18: 16.

Trumenba ${ }^{\circledR}$ vaccine. 2014. 'Wyeth Pharmaceuticals. Trumenba: prescribing information. [cited 2016 Mar 18]', http://www.fda.gov/downloads/BiologicsBloodVaccines/Vaccines/ApprovedProducts/UCM4211 39.pdf.

Wind, C. M., E. de Vries, M. F. Schim van der Loeff, M. S. van Rooijen, A. P. van Dam, W. H. B. Demczuk, I. Martin, and H. J. C. de Vries. 2017. 'Decreased Azithromycin Susceptibility of Neisseria gonorrhoeae Isolates in Patients Recently Treated with Azithromycin', Clin Infect Dis, 65: 37-45.

Yezli, S., A. Wilder-Smith, and A. A. Bin Saeed. 2016. 'Carriage of Neisseria meningitidis in the Hajj and Umrah mass gatherings', Int J Infect Dis, 47: 65-70.

Zhu, B., Z. Xu, P. Du, L. Xu, X. Sun, Y. Gao, and Z. Shao. 2015. 'Sequence Type 4821 Clonal Complex Serogroup B Neisseria meningitidis in China, 1978-2013', Emerg Infect Dis, 21: 925-32. 


\section{Table $\mathbf{1}$ (on next page)}

Epidemiological characterization of historical and emerging $N$. meningitidis isolates from military camps in Vietnam

'Isolates' metadata (year, clinical description, and location) were obtained from laboratory records of the Laboratory of Microbiology, Military Institute of Preventive Medicine, Hanoi. ${ }^{\text {b }}$ Molecular data was extracted from genomic sequence (NMB_VN2013, NMB_VN2014, NMB_VN2015) or laboratory records (other isolates). Strain designation was based on the previously recommended nomenclature (Jolley, Brehony, and Maiden 2007), comprising of serogroup, porA type (Px), fHbp type (Fx), and sequence type $(\mathrm{STX})(\text { clonal complex }(\mathrm{ccx}))^{\mathrm{c}}$ Unit 3 is geologically close to Unit $1,{ }^{d}$ Unit 4 is geologically close to Unit 2 
1 Table 1. Epidemiological characterization of historical and emerging N. meningitidis isolates

2 from military camps in Vietnam

\begin{tabular}{|c|c|c|c|c|c|}
\hline Isolate & Year ${ }^{a}$ & Description $^{\text {a }}$ & $\begin{array}{l}\text { Military } \\
\text { unit }^{\text {a }}\end{array}$ & $\begin{array}{l}\text { Sequence } \\
\text { type }(S T)^{b}\end{array}$ & Strain designation ${ }^{b}$ \\
\hline Khoa & 2008 & meningitis & Unit 2 & 1576 & B: P1.19,15-39: ST-1576 \\
\hline $37 \mathrm{C}$ & 2012 & carrier & Unit $3^{c}$ & 4821 & C: P1.20,2: ST-4821 (cc4821) \\
\hline $40 \mathrm{C}$ & 2012 & carrier & Unit $3^{c}$ & 4821 & C: P1.20,2: ST-4821 (cc4821) \\
\hline Bach & 2013 & meningitis & Unit 1 & 13455 & B: P1.19,15: ST-13455 \\
\hline NMB_VN2013 & 2013 & meningitis & Unit 2 & 1576 & B: P1.7-2,13: F4-6: ST-1576 \\
\hline 14072 & 2014 & carrier & Unit $4^{\mathrm{d}}$ & 13065 & B: P1.18,A: F18: ST-13065 \\
\hline 14075 & 2014 & carrier & Unit $4^{\mathrm{d}}$ & 13065 & B: P1. $\Delta, \Delta: \mathrm{ST}-13065$ \\
\hline 14089 & 2014 & carrier & Unit $4^{\mathrm{d}}$ & 13065 & B: P1. $\Delta, \Delta:$ F18: ST-13065 \\
\hline 14155 & 2014 & carrier & Unit 1 & 4821 & C: P1.20,2: ST-4821 (cc4821) \\
\hline 14156 & 2014 & carrier & Unit 1 & 4821 & C: P1.20,2: ST-4821 (cc4821) \\
\hline 14157 & 2014 & carrier & Unit 1 & 4821 & C: P1.20,2: F22: ST-4821 (cc4821) \\
\hline 14196 & 2014 & carrier & Unit 2 & 1576 & B: P1.7-2,13-2: ST-1576 \\
\hline NMB_VN2014 & 2014 & meningitis & Unit 1 & 13074 & B: P1.22-25,14-32: F4-6: ST-13074 \\
\hline 15020 & 2015 & carrier & Unit 1 & 1576 & B: P1.7-2,13-1: ST-1576 \\
\hline 1513 & 2015 & carrier & Unit 1 & 13056 & B: P1. $\Delta, \Delta:$ F18: ST-13065 \\
\hline 1523 & 2015 & carrier & Unit 1 & 1576 & B: P1.7-2,13-2: ST-1576 \\
\hline 1530 & 2015 & carrier & Unit 1 & 1576 & B: P1.22-25,14: ST-1576 \\
\hline 1533 & 2015 & carrier & Unit 1 & 1576 & B: P1.7-2,13: ST-1576 \\
\hline 1535 & 2015 & carrier & Unit 1 & 44 & B: P1.7-2,13-1: ST-44 (cc41/44) \\
\hline NMB_VN2015 & 2015 & meningitis & Unit 2 & 1576 & B: P1.22-25,14: F1-7: ST-1576 \\
\hline $1237 \bar{C}$ & 2016 & carrier & Unit $3^{c}$ & 4821 & C: P1.20,2: ST-4821 (cc4821) \\
\hline 16005 & 2016 & carrier & Unit 2 & 1576 & B: P1.22-25,14: ST-1576 \\
\hline 16016 & 2016 & carrier & Unit 2 & 1576 & B: P1.22-25,14: ST-1576 \\
\hline 16406 & 2016 & carrier & Unit 1 & 4821 & B: P1.7-2,14: ST-4821 (cc4821) \\
\hline 16408 & 2016 & carrier & Unit 1 & 4821 & B: P1.7-2,14: ST-4821 (cc4821) \\
\hline 16416 & 2016 & carrier & Unit 1 & 4821 & B: P1.7-2,14: F80: ST-4821 (cc4821) \\
\hline 17088 & 2017 & carrier & Unit 1 & 13074 & B: P1.22-25, $\Delta:$ ST-13074 \\
\hline 17090 & 2017 & carrier & Unit 1 & 13074 & $\mathrm{~B}: \mathrm{P} 1 . \Delta, \Delta: \mathrm{ST}-13074$ \\
\hline
\end{tabular}

a Isolates' metadata (year, clinical description, and location) were obtained from laboratory records of the Laboratory of Microbiology, Military Institute of Preventive Medicine, Hanoi.

${ }^{b}$ Molecular data was extracted from genomic sequence (NMB_VN2013, NMB_VN2014, NMB_VN2015) or laboratory records (other isolates). Strain designation was based on the previously recommended nomenclature (Jolley, Brehony, and Maiden 2007), comprising of serogroup, por $A$ type (Px), fHbp type (Fx), and sequence type (STx) (clonal complex (ccx))

${ }^{\mathrm{c}}$ Unit 3 is geologically close to Unit $1,{ }^{\mathrm{d}}$ Unit 4 is geologically close to Unit 2 


\section{Table 2 (on next page)}

Allelic profiles of antibiotic resistant genes ${ }^{a}$ and antibiotic susceptibility of the Vietnamese isolates ${ }^{b}$

${ }^{\text {a }}$ An allele number was assigned to each locus based on its DNA sequence using PubMLST database ( https://pubmlst.org/ ) ( Jolley and Maiden 2010 ) ${ }^{b}$ Antibiotic susceptibility of isolates was examined using E-test strip (bioMerieux, France) and interpreted according to CLSI 2018 breakpoints ( CLSI 2018 ) . NA*: new, unassigned alleles identical at said locus; I: Intermediate, S: Susceptible, R: Resistance. Numbers below each susceptibility interpretation indicate MIC $(\mu \mathrm{g} / \mathrm{ml})$ values. 
1 Table 2. Allelic profiles of antibiotic resistant genes ${ }^{a}$ and antibiotic susceptibility of the

2 Vietnamese isolates ${ }^{b}$

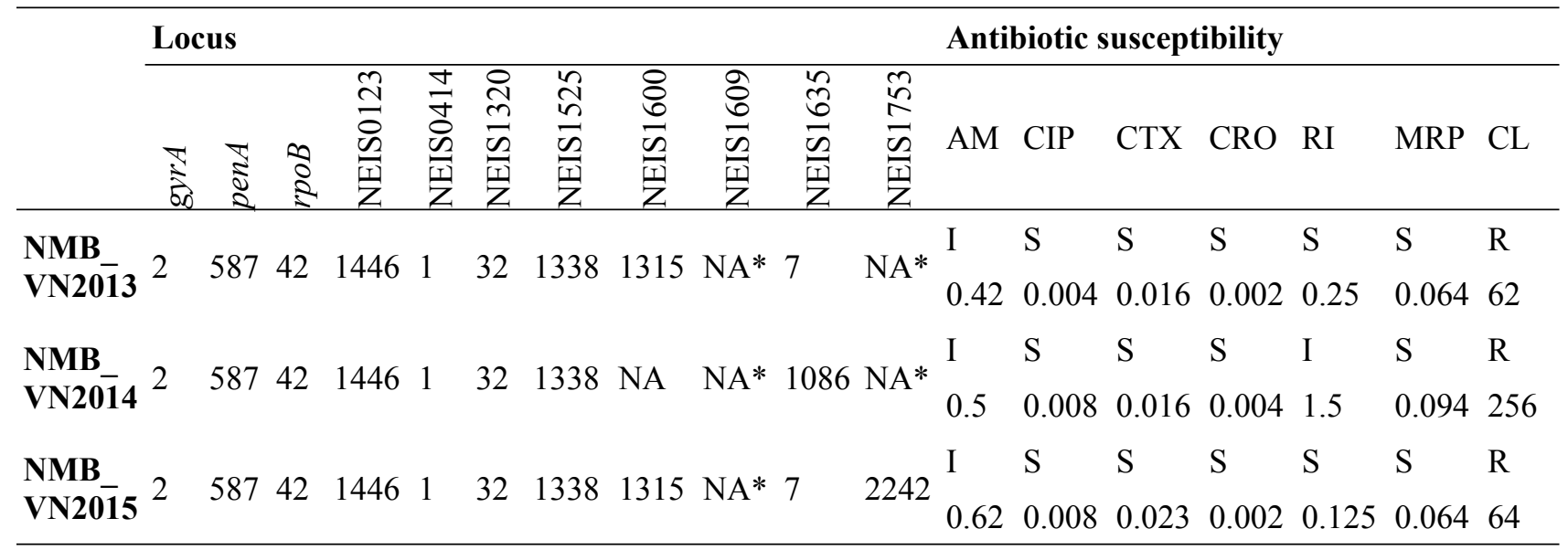

3 a An allele number was assigned to each locus based on its DNA sequence using PubMLST

4 database (https://pubmlst.org/) (Jolley and Maiden 2010)

5 b Antibiotic susceptibility of isolates was examined using E-test strip (bioMerieux, France) and

6 interpreted according to CLSI 2018 breakpoints (CLSI 2018).

7 NA*: new, unassigned alleles identical at said locus; I: Intermediate, S: Susceptible, R:

8 Resistance. Numbers below each susceptibility interpretation indicate MIC $(\mu \mathrm{g} / \mathrm{ml})$ values. 


\section{Table 3 (on next page)}

Antigenic profile of Vietnamese and Southeast Asian isolates, with middle line separate the chloramphenicol-resistant (above) and susceptible (below) isolates

${ }^{a}$ Sequence type (ST) and clonal complex (cc) determined by the sequence of seven housekeeping genes $(a b c Z, a d k, a r o E$, fumC, $g d h, p d h C$, and $p g m){ }^{b}$ Allele number assigned to each locus based on its DNA (porA) or protein ( $f H b p, n h b A$, and fet $A$ ) sequences ${ }^{a, b}$ Analyses was performed using PubMLST database ( https://pubmlst.org/ ) ( Jolley and Maiden 2010 ) 
1 Table 3. Antigenic profile of Vietnamese and Southeast Asian isolates, with middle line separate 2 the chloramphenicol-resistant (above) and susceptible (below) isolates

\begin{tabular}{|c|c|c|c|c|c|c|}
\hline \multirow{3}{*}{ Isolate } & \multirow{3}{*}{$\begin{array}{l}\text { Sequence type } \\
\text { and clonal } \\
\text { complex } \\
(\mathrm{ST}(\mathrm{cc}))^{\mathrm{a}}\end{array}$} & \multicolumn{5}{|c|}{ Antigenic profile ${ }^{b}$} \\
\hline & & \multicolumn{2}{|c|}{$\operatorname{por} A$} & \multirow{2}{*}{$\begin{array}{l}\text { fHbp } \\
\text { Peptide }\end{array}$} & \multirow{2}{*}{$\begin{array}{l}n h b A \\
\text { Peptide }\end{array}$} & \multirow{2}{*}{ fet $A$} \\
\hline & & VR1 & VR2 & & & \\
\hline NMB_VN2013 & 1576 & $7-2$ & 13 & 31 & 16 & $4-6$ \\
\hline NMB_VN2014 & 13074 & $22-25$ & $14-32$ & 31 & 16 & $4-6$ \\
\hline NMB_VN2015 & 1576 & $22-25$ & 14 & 31 & 16 & $1-7$ \\
\hline NM01- & 14487 & 19 & 15 & 283 & 16 & $1-20$ \\
\hline NM11 & 14496 & 19 & 15 & 31 & - & $3-7$ \\
\hline NM12 & 1576 & $19-1$ & $15-31$ & 31 & 16 & $4-6$ \\
\hline NM13 & 1576 & 19 & 15 & - & 16 & $5-88$ \\
\hline NM16 & 1576 & 19 & 15 & 31 & - & - \\
\hline NM18 & 1576 & 19 & 15 & 1035 & 16 & $3-31$ \\
\hline NM20 & 11005 & 19 & $15-39$ & 31 & 16 & $5-135$ \\
\hline NM25 & 1576 & 19 & 15 & 31 & 16 & - \\
\hline NM14 & $1145(\operatorname{cc} 41 / 44)$ & $7-2$ & 4 & 14 & 2 & $1-20$ \\
\hline NM15 & $41(\operatorname{cc} 41 / 44)$ & $7-2$ & 4 & 14 & 2 & $1-19$ \\
\hline NM19 & $14503(\operatorname{cc} 4821)$ & 20 & $23-7$ & 141 & 669 & - \\
\hline NM21 & 12811 & $12-1$ & $13-1$ & 18 & 945 & $1-19$ \\
\hline NM23 & 14507 & 22 & $23-1$ & - & 21 & $4-21$ \\
\hline NM03 & $14488(\operatorname{cc} 41 / 44)$ & $7-2$ & 4 & 14 & 2 & $1-49$ \\
\hline NM04 & 14489 & $22-15$ & - & 5 & - & - \\
\hline NM06 & $32(\operatorname{cc} 32)$ & 18 & - & 101 & - & $1-21$ \\
\hline NM07 & 3256 & $7-1$ & - & 24 & 1086 & $3-1$ \\
\hline NM09 & 5604 & $22-1$ & 26 & - & 1068 & $3-2$ \\
\hline
\end{tabular}

3 a Sequence type (ST) and clonal complex (cc) determined by the sequence of seven house-

4 keeping genes ( $a b c Z, a d k, a r o E, f u m C, g d h, p d h C$, and $p g m)$

5 b Allele number assigned to each locus based on its DNA (porA) or protein ( $f H b p, n h b A$, and 6 fet $A$ ) sequences

7 a, b Analyses was performed using PubMLST database (https://pubmlst.org/) (Jolley and Maiden 8 2010) 


\section{Table 4 (on next page)}

Diversity of virulent determining factors from genome sequence of the Vietnamese isolates

${ }^{\text {a }}$ Allele numbers assigned to each locus based on DNA sequence using PubMLST database ( https://pubmlst.org/ ) ( Jolley and Maiden 2010 ) ${ }^{\mathrm{b}}$ Comparison result at $\mathrm{cnl}$ (capsule null locus) was omitted since all isolates were capsulated ${ }^{\mathrm{C}}$ Allele numbers assigned to each locus based on protein sequence using PubMLST NA: new, unassigned alleles; NA*: new, unassigned alleles identical at said locus 
1 Table 4. Diversity of virulent determining factors from genome sequence of the Vietnamese

2 isolates

Locus Gene Product

\section{Capsular gene cluster ${ }^{a, b}$}

NEIS0046 $r f b A \quad$ glucose-1-phosphate thymidylyltransferase NA NA NA

NEIS0054 css $\quad$ N-acetylglucosamine-6-P 2-epimerase NA* NA* 113

NEIS0067 ctrF capsule translocation 4 NA* NA*

Maf-toxin genomic island ${ }^{a}$

$\begin{array}{llllll}\text { NEIS1788 } & \text { anmK } & \text { anhydro-N-acetylmuramic acid kinase } & 25 & 25 & \text { NA } \\ \text { NEIS1795 } & \text { mafI }_{\text {o2MGI-2 }} & \text { MafI immunity protein } & 4 ; 186 & 4 & 4 ; 186 \\ \text { NEIS2083 } & \text { maf }_{M G I-3} & \text { MafA3 lipoprotein } & 252 & 252 & 252 ; 890 \\ \text { NEIS2084 } & \text { mafB }_{M G I-3} & \text { MafB3 toxin protein } & 31 & \text { NA } & 31\end{array}$

Outer membrane vesicle (OMV)peptide ${ }^{c}$

$\begin{array}{lllll}\text { NEISp0653 - } & \text { Competence lipoprotein } & \text { NA* } & \text { NA* } & 1 \\ \text { NEISp0275 - } & \text { Organic solvent tolerance protein } & \text { NA* } & \text { NA } & \text { NA* } \\ \text { NEISp0923 - } & \begin{array}{l}\text { Antioxidant AhpC TSA family } \\ \text { glutaredoxin }\end{array} & 2 & 2 & \text { NA } \\ \text { NEISp1364 - } & \text { Outer membrane protein PorA } & 155 & \text { NA } & \text { NA } \\ \text { NEISp1687 - } & \text { Phospholipase A1 } & 254 & 254 & \text { NA } \\ \text { NEISp1690 - } & \text { Transferrin-binding protein 1 } & \text { NA* } & \text { NA* } & \text { NA } \\ \text { NEISp1963 - } & \text { Iron-regulated outer membrane protein } & \text { NA* } & \text { NA* } & \text { NA }\end{array}$

\section{Pilin genes $^{a}$}

\begin{tabular}{llllll} 
NEIS0020 & pilB/msrAB & peptide methionine sulfoxide reductase & 379 & 11 & NA \\
NeIS0021 & pilA/fts $Y$ & $\begin{array}{l}\text { MsrA/MsrB } \\
\text { probable signal recognition particle protein NA }\end{array}$ & 1778 & 1978 \\
NEIS0036 & pilT1 & type IV pilus retraction ATPase PilT & 200 & 11 & 11 \\
NEIS0210 & pilE & PilE & NA & NA & NA \\
NEIS0213 & pglA & pilin glycosyltransferase & NA & 1053 & 1053 \\
NEIS0830 & pilK & type IV biogenesis protein & 1905 & 1618 & 1618 \\
pilS & pilS cassette - & NA & NA & NA \\
\hline
\end{tabular}

3 a Allele numbers assigned to each locus based on DNA sequence using PubMLST database

4 (https://pubmlst.org/) (Jolley and Maiden 2010)

5 b Comparison result at $c h l$ (capsule null locus) was omitted since all isolates were capsulated

$6{ }^{c}$ Allele numbers assigned to each locus based on protein sequence using PubMLST

7 NA: new, unassigned alleles; NA*: new, unassigned alleles identical at said locus 


\section{Figure 1}

Genealogical relationship of three Vietnamese isolates with the global $108 \mathrm{~N}$. meningitidis isolate collection (Bratcher et al. 2014) (A) and the 18 Southeast Asian isolates (Batty et al. 2019) (B) revealed by a 1605-locus core genome comparison.

These isolates represent the major hyper-invasive lineages/sub-lineages recorded worldwide in the last 70 years and the available genomic information from the Southeast Asia region, respectively. The Vietnamese isolates were highlighted by green nodes and labels in both trees, and major lineages/sub-lineages were indicated by color: navy - ST41 sub-lineage (ten isolates), blue - ST44 sub-lineage (four isolates), pink - Southeast Asian chloramphenicol resistant lineage (eight isolates), purple - Southeast Asian chloramphenicol-susceptible isolates (ten isolates). Together, ST41 and ST44 sub-lineage make up Lineage 3 (ST-41/44 clonal complex).

A

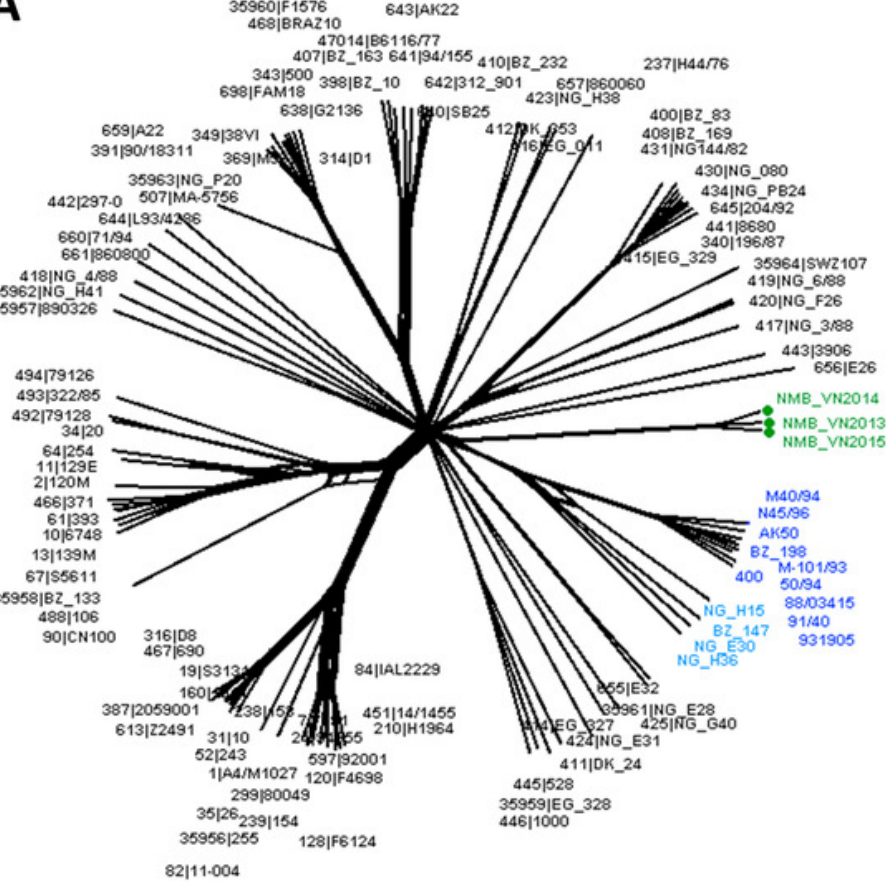

B

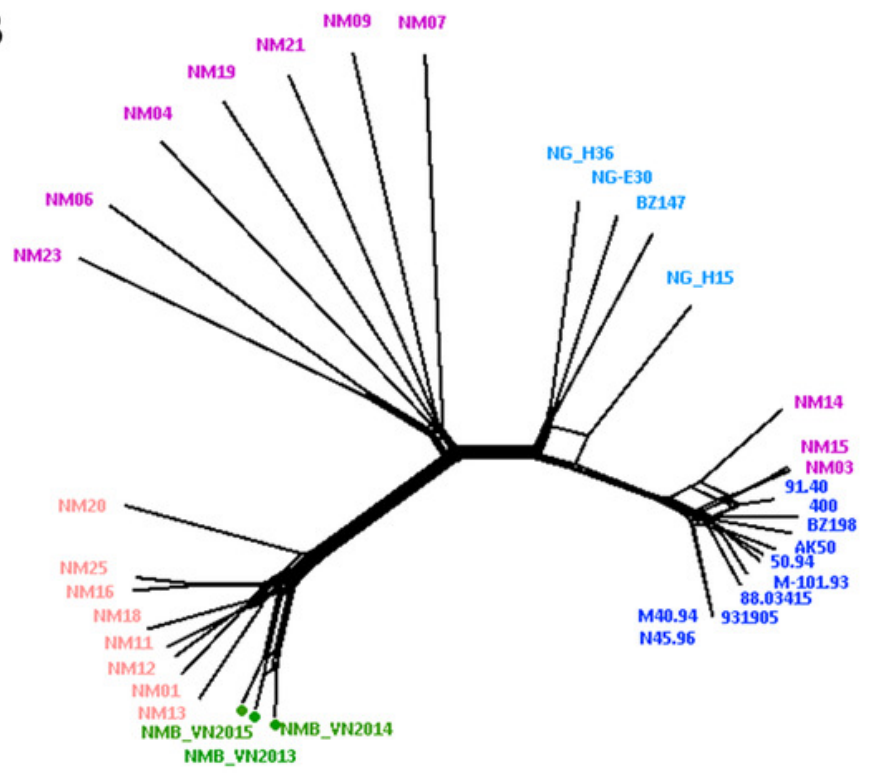

